Journal of Engineering and Applied Sciences 15 (2): 636-642, 2020

ISSN: 1816-949X

(C) Medwell Journals, 2020

\title{
Fall Detection with Support Vector Machine for Elderly Care using Pressure Sensor Grid
}

\author{
Viknesh Kumar, Boon-Chin Yeo and Way-Soong Lim \\ Faculty of Engineering and Technology, Multimedia University Melaka, Malacca, Malaysia
}

\begin{abstract}
Generally, falling is prevalent in elderly of age 65 and above due to age-related biological changes. Falls can be life-threatening if noticed late. Hence, a fall detection and surveillance device was developed to monitor elderly living alone. The primary objective is to build a pressure sensitive mat capable of detecting fall through image processing. The subjects tested resembled the stature of elderly. The accuracy of the system in detecting fall is $93 \%$ while the accuracies for the other postures such as standing and sitting yield 93.5 and $81.5 \%$, respectively.
\end{abstract}

Key words: Fall detection, pressure sensor, support vector machine, elderly care, postures, objective

\section{INTRODUCTION}

The population of elderly is ever growing both locally and around the globe. The proportion of population aged 60 years and older in Malaysia was 8\% in 2010. The percentage will continue to increase to $14 \%$ around 2030 in which Malaysia will be an aging nation at the time (Abeykoon et al., 2017). Hence, a need for a proper tracking and fall detection tool is high to reduce hazard involving elderly whom are living alone.

According to the World Health Organization (WHO), about $28 \%-35 \%$ of elderly aged 65 and $32 \%-42 \%$ elderly over 70 years of age experience fall each year. The factors that affect increase in fall is age and frailty (WHO., 2008). In other terms, geriatric syndrome in elderly contributes to high morbidity and mortality among elderly. Hence, falling increases exponentially with age-related biological changes which leads to high incidence of falls (Dhargave and Sendhilkumar, 2016). If no preventive measures are made in immediate future, the toll of injuries caused by falling will increase up to a $100 \%$ higher in 2030 (Romli et al., 2016).

Falls are common in areas such as bathrooms which is often considered private sector of home which lacks monitoring. Hence, this results in a blind spot and increases risks of injuries and death if falls in such areas are identified late.

Fall identification devices sold in markets come in two categories; wearables and vision. Wearable devices rely on users to carry them about as tags or other creative that can be often neglected indoors (Hwang and Pan, 2016). Vision systems, though reliable, it is unethical to place them in private areas where privacy is a concern. Most information channeled from cameras should not be available to human operators and can be deemed a privacy intrusion (Kerr and Schyndel, 2014). Hence, an alternative system is to be devised in this project.
Thus, in this project the electronic mat is constructed to observe the movement of resident within the household. This tracking method allows to observe and alert users in cases of emergency. This tracking device is useful in monitoring the movement of old folks without invading their personal space. This device is made indoor friendly.

\section{Literature review}

Technologies for fall detection: Devices currently available for identifying falls can be sectored into wearable devices and non-wearables. Analysis was done to identify the accuracy of fall detection as well as the pros and cons of each system.

A wearable device could detect a falling impact accurately. According to survey by Chaudhuri et al. (2014), about $19 \%$ of the wearable projects utilized elderly to test their devices under a controlled environment and only $7.1 \%$ of the projects were done in real world settings. The remainder studies relied on healthy young test subjects. About 35 of the projects studied used a single fall detection device while 4 used 2 separate fall detection devices while another 4 study used 3 separate devices. Studies related to wearable devices usually require the test subjects to wear the sensors on chest, waist, head, arms, hands and feet.

According to Chaudhuri et al. (2014), wearable devices that are targeted at the trunk of the body has 97.5 and $96.9 \%$ range for median sensitivity and median specificity, respectively. Systems that involve multiple sensors were carrying median sensitivity and median specificity of 93.4 and 99.8\%, respectively. Finally, devices worn around the areas of arm, hands, ears or feet had a much lower median of sensitivity and specificity which are 81.5 and $83 \%$. Though wearables do carry a disadvantage where the user has to remember to wear it 
prior to falling event. Choosing to wear the device all the time is not a viable option as it may be difficult indoors. Another drawback to wearable devices is the need of battery as power supply and frequent false alarms due to jitters.

Vision systems and motion detectors are classified as non-wearable devices which could monitor the user without affecting the user's movement. Non-wearable devices were often set in rooms which test subjects would walk around or stay for some period of time. However, no studies using this system had mentioned of tests involving elderly. Motion detectors are usually infrared sensors that detect motion while vision system provides series of live images. Most of the studies use single camera detection, although, some studies use multiple camera networks (Auvinet et al., 2008; 2011). Most camera systems were stand-alone; however, introducing reflective sensors improve the accuracy. The median accuracy for cameras was listed at a range of $96.6 \%$ while the median sensitivity and specificity falls at 93 and 98.5\%, respectively. However, cameras are prone to privacy intrusion as the systems record too much information for analysis. Both the devices sometimes suffer from occlusion or involuntary blocking by objects in the room.

The survey shows an insight to the preference of elderly. Though elderly prefer to live independently and do not prefer fall detection devices due to annoyance of false alarm; the users are open to system that could give them a greater sense of security. Hence, there is a need in the area of work to implement an accurate device that is able to detect fall and at the same time unobtrusive (Brownsell and Hawley, 2004). Our previous works implement feature-based fall detection with pressure sensor mat for elderly care (Kumar et al., 2018).

Pressure sensor mat: As for implementing an electronic mat as an alternative fall detection, various designs were studied. Analysis on the hardware and software construction of these mats were done to observe the possible pros and cons (Chaudhuri et al., 2014; Klenk et al., 2011; Wilding et al., 2013).

Among research findings, uses a sensor mat in detecting pressure distribution use modules of transduction module. The system is interlinked by sub-modules that branch to form a $32 \times 24$ matrix of pressure sensors. The modules are composed of slaves and master boards. Each sub modules have a dedicated slave board attached to them. The boards aid in acquiring signals from the sensors. The signals translated using a microcontroller and processed on a user interface. The user interface can identify the shape of object placed on the mat.

Pressure sensing mat can be used in tele-homecare system (Saenz-Cogollo et al., 2016). A piece of fabric treated using polymer solutions at all cross points. At top and bottom part of the fabric is stitched with conductive threads to form a conductive platform. To avoid short circuit, measures are taken while stitching, so, the conductive paths never meet. Data acquisition is done using Arduino Yun. With 8-bit resolution, the matrix size detected is up to $32 \times 32$ array. The user can access the system anywhere and anytime for monitoring.

The research by Kim et al. (2015) uses similar pressure sensor mat which is formed with a mesh of piezo-resistive fabric and conductive threads. Rows and columns are made by sewing silver plated threads. A zig-bee module is used to process the data. A User Interface at receives the data and translates the pressure values into image. The user interface collects data via internet from one mat to another. A decision-making server is used to analyze the environment based on outputs from the mat sensors.

For fall detection, the pressure sensor mat is generally, big. A module-based mat might not be enough without joining multiple mats. In other words, a single mat is at its peak in number of conductive array and resized without the user having buy or build similar mats to be attached in series. Hence, a sizable mat design is needed to be constructed at low cost.

Meanwhile, stitching of conductive threads for pressure sensor mat development can be time-consuming when constructing a mat of large scale. Hence, an alternative approach is needed to accommodate productivity.

Some of the studies require the analysis to be done remotely at the server side. The drawback is that if the terminals are cut due to poor internet connectivity, the processing of the images will not take place and disrupt detection at crucial moments. In this study, the fall detection algorithm is resided at the computer connected to the sensor through serial communication.

Support Vector Machine (SVM): Feature extraction and computation using Support Vector Machine (SVM) machine learning is applied in detecting fall events. Reasons for choosing the supervised machine learning method is that it has been tested with various fall identification devices and has given a good yield in detecting fall.

A study has used various machine learning methods including SVM. The device that was tested on is a wearable device that the user wears at the trunk of the body. The study concludes that in comparison between the performance on the same dataset between various machine learning data including SVM shows the system could achieve accuracies above 95\% (Ozdemir and Barshan, 2014). The test that was done to conclude the accuracy was a repeatability test of results over 10 times which indicates the researcher of the robustness of the classifiers. 
Another study also a wearable based analysis uses SVM for fall identification. The yield for this study is said to be much higher than previous study which could be attributed to the large number of features being extracted to conclude fall, amounting to 6 feature extraction. The results show an accuracy of $98.7 \%$ (Shibuya et al., 2015).

The reseacrh by Yu et al. (2012) applies SVM on vision based fall identification system. This system recognizes various postures including lying, squatting, sitting and standing. The study reports $97.08 \%$ fall identification accuracy while using SVM.

\section{MATERIALS AND METHODS}

Hardware design: The hardware is a mesh of piezo-resistive material and conductive grid. The hardware is connected to a microcontroller (Arduino Mega) network to convert pressure data into image. The hardware is constructed at a size of $1.36 \times 1.36 \mathrm{~m}$.

The resolution of conductive grid used is $48 \times 48$; denotes 48 rows as shown in Fig. 1 a and 48 columns as shown in Fig. 1 b. The unique mesh of conductive grids and piezo-resistive material as displayed in Fig. 2 form an equivalent of 2304 pressure sensors.

The resulting hardware is as shown in Fig. 3. The choice of fabric for the mat is based on user's selection. In this experiment, we had opted for a PVC based fabric as it is waterproof and dust-proof; allowed testing crucial private areas of home such as bathrooms and bedrooms where elderlies are susceptible to falls.

Fall detection with SVM: Image based data collection is chosen as a viable option for identification of falls because as per study suggests; some elderly may experience falls as random and unpredictable events while most have a distinct falling pattern which indicates potential risk factors and can be used to predict adverse outcomes.

Knowing the patterns of fall and the risks and outcomes due to falling may help clinical personnel to plan preventive measures and provide medical assistance to the affected patient ( $\mathrm{Lu}$ and Chu, 2018).

Image data gathered can help recognize patterns and activities prior falling event for functional assessment. Functional assessment is the recognition of elderly's physical attribute that contributes to risk of falling. This means the system will record multiple data pre-fall and store based on different postures to identify balance impairment by clinicians during rehabilitation and prevent intrinsic risks that contribute to falling of elderly at home. The postures which the system recognizes are standing, sitting and lying down or fall. The postures are captured based on pressure exertion of user on the mat. The image is plotted using OpenTK library. Fig.4 -6 show the images of sitting, standing and lying down or fall images captured, respectively.

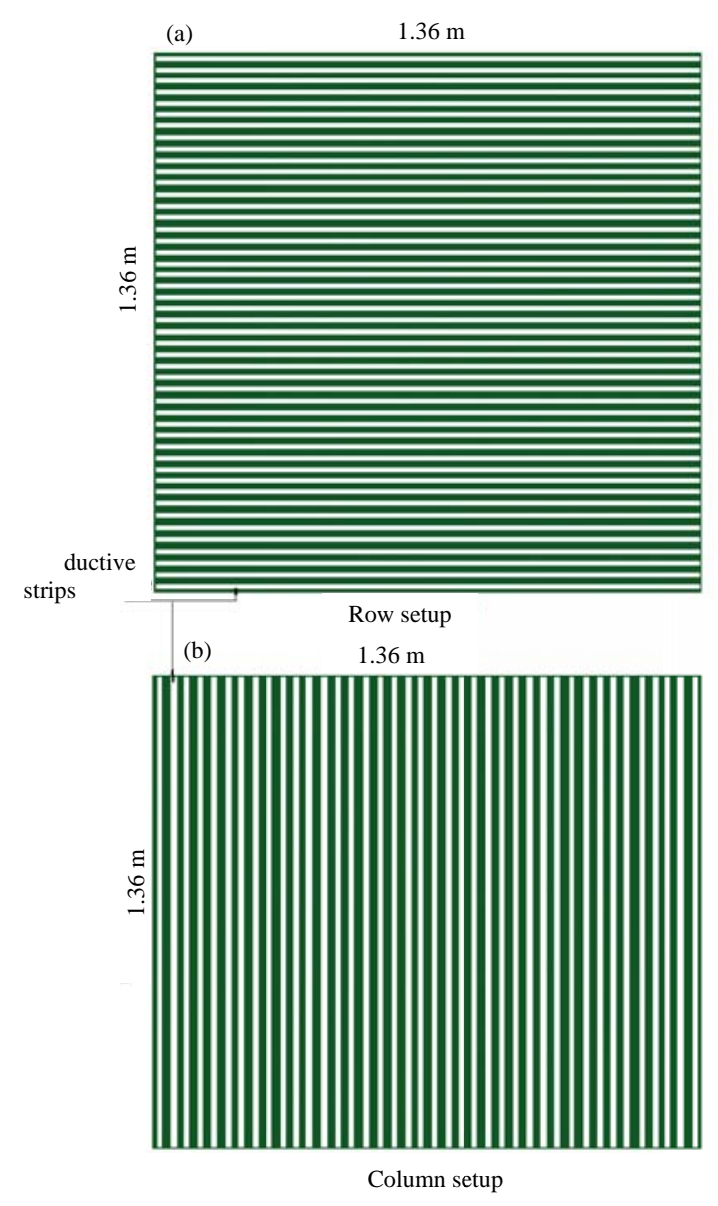

Fig. 1: Setup of conductive layers on the pressure mat (a) A row setup and (b) A column setup

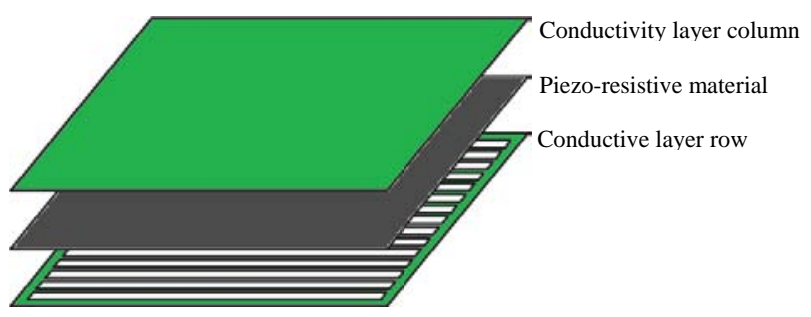

Fig. 2: Sandwich-layering structure for pressure sensor mat

Pseudo-colors are included to differentiate pressure at each cross section of the grid. The transition of colors is from blue which denotes lowest pressure to red which denotes the highest pressure exerted on the mat. To differentiate the images based on posture and reduce false alarms, machine learning method Support Vector Machine (SVM) was chosen.

In this study, SVM is used as many previous works has reported high accuracy for introducing SVM for fall 


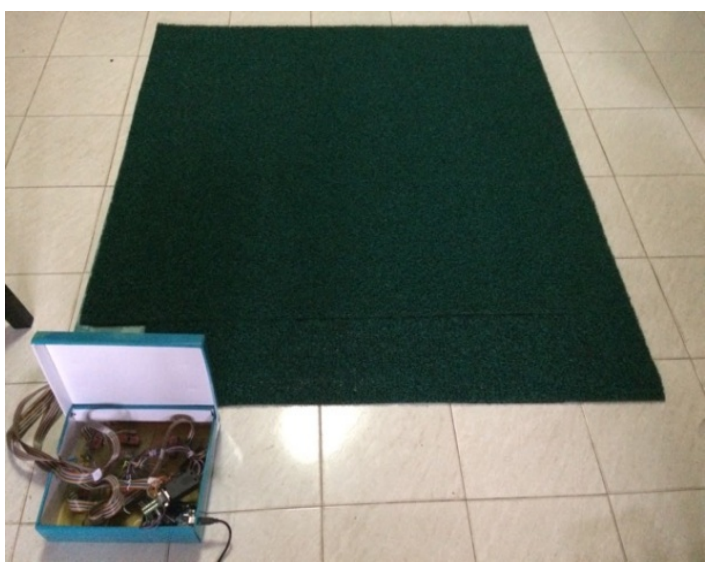

Fig. 3: The pressure sensitive mat is made of PVC fabric and is connected to a controller box to transmit signal to PC for analysis

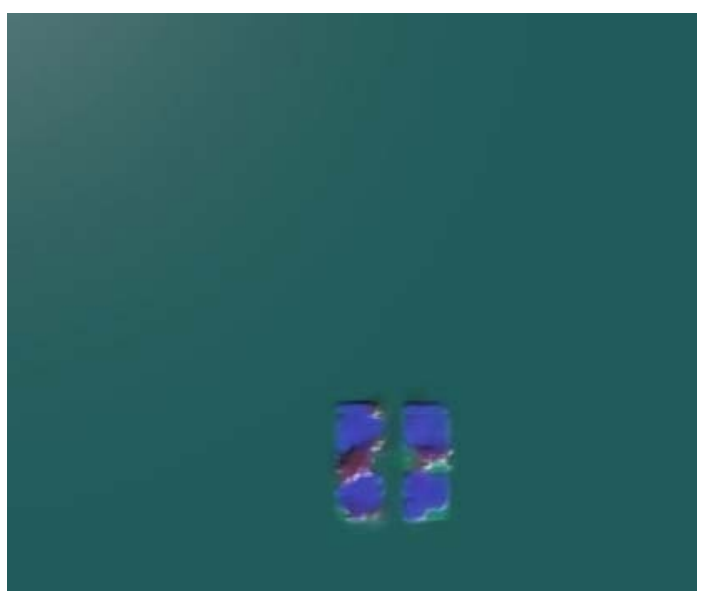

Fig. 4: The pressure sensitive mat records standing posture

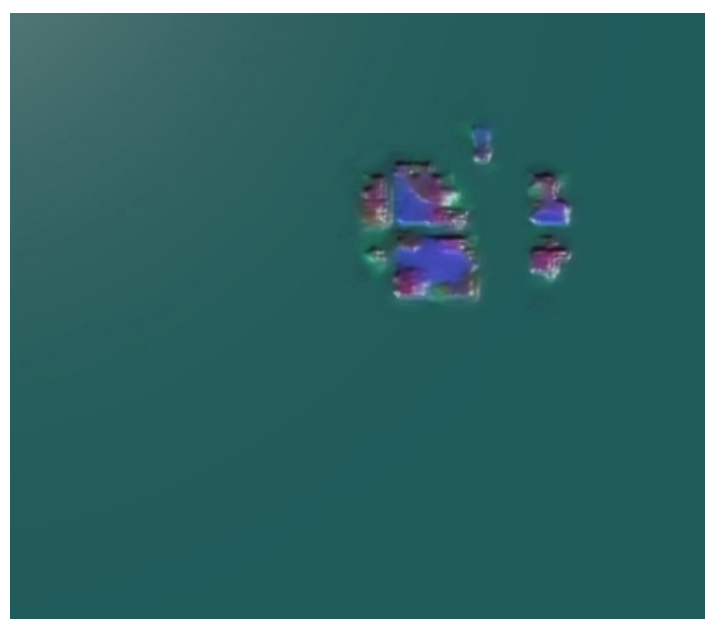

Fig. 5. The pressure sensitive mat records sitting posture

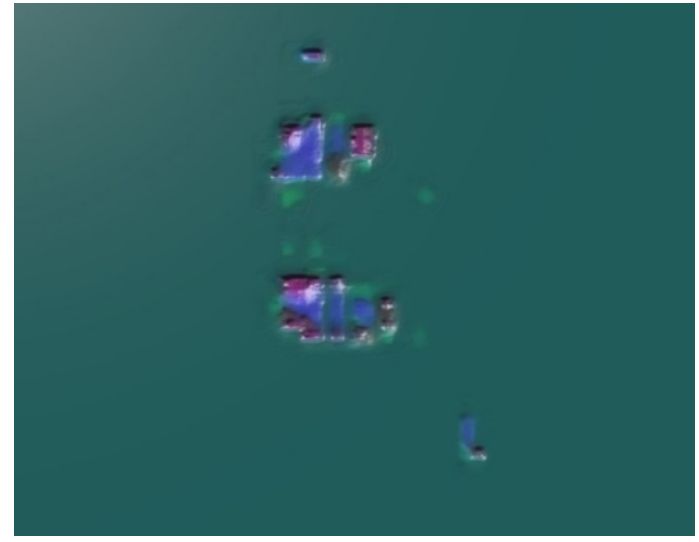

Fig. 6: The pressure sensitive mat records lying down or fall

detection. A set of data was gathered and fed to the computation to allow detection of different postures such as standing, sitting and fall. This way it was easier for the software to distinguish different postures from fall while reducing false alarm rate.

The form of SVM used in this project is a Multiclass-SVM (Xu et al., 2017) is used to classify the images generated from the pressure sensor mat. Prior to multi-class SVM classification, images are normalized. This is done to preserve quality of inspection by applying histogram stretching to enhance the details in the image (Dey et al., 2016). The reason for applying histogram is because image is a set of pixels of many colors. The pixel's color is described by several values represented by RGB values. Using this notion, we analyze the area of coverage by pressure by distinguishing difference between the color of background and areas where pressure is applied. Before normalization let denote the image as shown in Eq. 1 Fig. 6:

$$
\mathrm{I}=\left\{\mathrm{X} \subseteq \mathrm{R}^{\mathrm{n}}\right\} \rightarrow\{\text { Min }, \ldots, \operatorname{Max}\}
$$

During pre-normalization, histogram or contrast stretching is done to enhance the details on the image. Then, the image undergoes normalization process as shown in Eq. 2. The normalization process will brighten the image regions where pressure is applied to the sensor mat. At the same time, the other regions are remained dim. During normalization, the values of and are set to 1 and 0.34 , respectively and are the maximum and minimum allowable pixel intensity values, respectively:

$$
\mathrm{I}_{\mathrm{N}}=(\text { newMax-newMin }) \cdot \frac{1}{1+\exp \left(\frac{\mathrm{I}-\beta}{\mathrm{a}}\right)}+\text { newMin }
$$

Data collection: The research is intended for elderly of aged 65 and above. Due to physical inhibit of elderly, 
younger test subjects with stature resembling the elderly were chosen. The test subjects are chosen with particular height and weight ranges which are 160 and $170 \mathrm{~cm}$ in height and 60-70 kg in weight, respectively. The weight and height assumptions are based on study of average elderly weight and height range in Asians (Darnis et al., 2012; Igual et al., 2013).

The reason in which a controlled weight and height range is accounted is due to biological changes in old age. During aging, the cartilage between joints of an elderly are worn out due to osteoporosis that shrinks the size of spinal column. This changes height of an average person to fit in the category of height assumption made.

The assumption of weight is made based on varying weight loss in men and woman. Men often gain weight until about age 55. They tend to lose weight later in life. This may be related the drop-in testosterone hormone. Women usually gain weight until age 65. Weight loss only happens beyond the stipulated age. Weight loss happens partly due to fat being replaced with lean muscle tissue.

This experiment was carried out with 10 subjects of different age, height, weight and gender. Common postures such as sitting, standing and lie down or fall on the mat were performed by volunteers and the accuracy of the system in differentiating data was recorded. The experiment was done in a home to mimic real world application. Each subject contributed 20 samples for each posture. The samples are then fed to the system for supervised teaching and the experiment was repeated again to identify the accuracy of the system. The observation was recorded.

\section{RESULTS AND DISCUSSION}

The accuracy of detecting 3 postures; sitting, standing and fall were observed and recorded. A total of 600 samples; 200 samples for each posture were taken and fed to the system to teach the software to identify different postures. The experiment was repeated by seeking the test subjects to perform random postures on the mat to monitor the accuracy. Table 1 displays the accuracy of the system to estimate the model is done by using system identification.

The percentage of detecting fall is $93 \%$. The mat could detect falls at high accuracy. Standing posture leans towards 93.5\% accuracy and the sitting posture is at $81.5 \%$. The detection of different postures happened at real-time as the system inspects 2 frames per second.

Analysis show the reasons for false alarms despite feasibly high fall identification accuracy is due to complexity of classifying postures such as sitting from the postures performed during a fall. Some of these sitting postures have similar area coverage and pressure distribution to that results from falling, hence, a false alarm is generated. A sample data for sitting posture that looks similar to a fall data, as shown in Fig. 7 and 8.

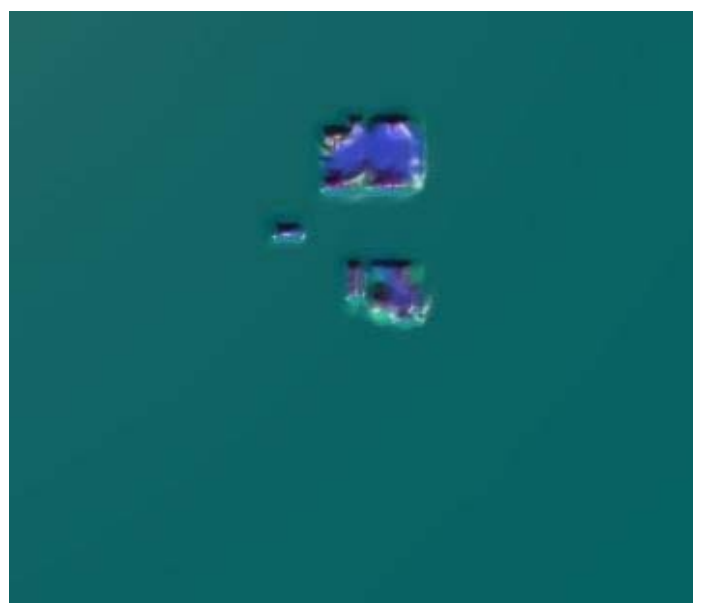

Fig. 7: Data for sitting posture which is similar to falling

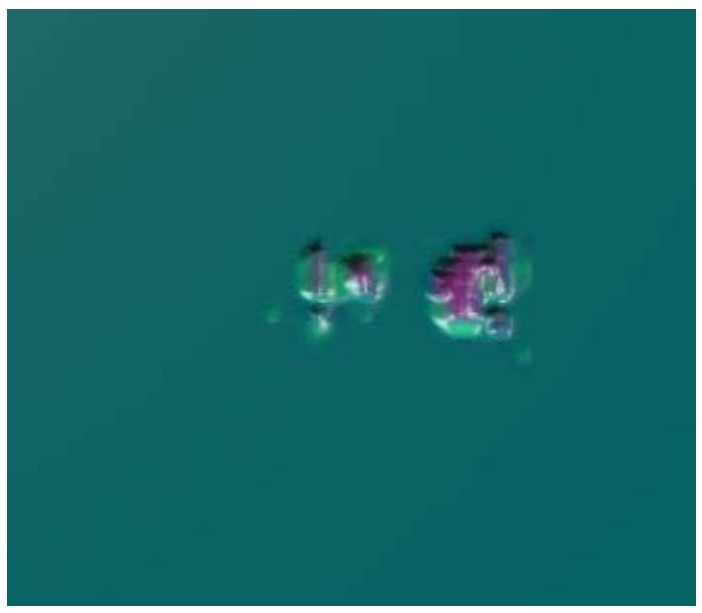

Fig. 8: Data for fall which is similar to sitting posture

\begin{tabular}{|c|c|c|c|}
\hline \multirow{2}{*}{$\begin{array}{l}\text { Posture } \\
\text { performed }\end{array}$} & \multicolumn{3}{|c|}{ Posture detected by SVM Software } \\
\hline & Standing & Sitting & Fall \\
\hline Standing & $187 / 200$ & $13 / 200$ & $0 / 200$ \\
\hline Sitting & $15 / 200$ & $163 / 200$ & $22 / 200$ \\
\hline Fall & $1 / 200$ & $14 / 200$ & $186 / 200$ \\
\hline
\end{tabular}

Figure 7 is data that was captured when a volunteer performed a sitting posture termed as side-saddling. The data in Fig. 7 which was used to train the SVM system carried the same weight in multi-SVM classification to Fig. 8, a fall data that was captured when a volunteer decided to fall in a curled fetal position while testing the accuracy of the system upon training. The size and impact done of the two data appear similar in nature causing an anomaly while classification.

Classification issues that arise which contributes to false alarm are mainly due to the impedance in imagination when it comes to predicting and performing 
different styles of sitting, standing and fall to be trained into the machine learning system. Such data classification distortion can only be significantly reduced through mass data collection.

However, the current experiment was a success as it is still able to detect falls with less false alarm despite limited data available for training. A new method of fall detection has been devised.

\section{CONCLUSION}

With rising global elderly population, there is an increased necessity for fall detection devices. By eliminating the need for vision systems which are a privacy concern and wearable devices that are hassle for elderly to carry about indoors; this study explores fall identification system through image processing for high accuracy. A new fall identification device is devised for the benefit of elderly.

The device made is a viable replacement for other fall detection-based devices available. It is able to detect falls with 93\% accuracy making it a nominal option to monitor elderly at home.

\section{LIMITATIONS}

The subjects tested, though resembling the stature of elderly, there are still biological impedance and differences to be explored. Hence, a field test involving elderly will be done.

\section{ACKNOWLEDGEMENT}

We thank the institution Multimedia University for providing a platform for research and analysis. The expertise and insight given to us greatly enhanced this research.

We thank volunteers for contributing data intended for this research. Samples collected vastly improved the accuracy of the system for desirable yield in detection of fall. We express our gratitude to family members for their wisdom and guidance in making this project successful.

\section{REFERENCES}

Abeykoon, A., N. Arifin, T.N. Peng, C. Kannarath and A.C.B. Naraval, 2017. Ageing: demographic transition, policy and programmatic response. International Council on Management of Population Programmes (ICOMP), Selangor, Malaysia. http://site.icomp.org.my/Clients/icomp1/icomp-ippfageing_case-studies-for-website.pdf.

Auvinet, E., F. Multon, A.A. Saint, J. Rousseau and J. Meunier, 2011. Fall detection with multiple cameras: An occlusion-resistant method based on 3-d silhouette vertical distribution. IEEE. Trans. Inf. Technol. Biomed., 15: 290-300.
Auvinet, E., L. Reveret, A. St-Arnaud, J. Rousseau and J. Meunier, 2008. Fall detection using multiple cameras. Proceedings of the 2008 30th Annual International Conference on IEEE Engineering in Medicine and Biology Society, Augut 20-25, 2008, IEEE, Vancouver, Canada, pp: 2554-2557.

Brownsell, S. and M. Hawley, 2004. Fall detectors: Do they work or reduce the fear of falling?. Hous. Care Support, 7: 18-24.

Chaudhuri, S., H. Thompson and G. Demiris, 2014. Fall detection devices and their use with older adults: A systematic review. J. Geriatr. Phys. Ther., 37: 178-196.

Darnis, S., N. Fareau, C.E. Corallo, S. Poole, M.J. Dooley and A.C. Cheng, 2012. Estimation of body weight in hospitalized patients. QJM. Int. J. Med., 105: 769-774.

Dey, M., B. Raman and M. Verma, 2016. A novel colour-and texture-based image retrieval technique using multi-resolution local extrema peak valley pattern and RGB colour histogram. Pattern Anal. Applic., 19: 1159-1179.

Dhargave, P. and R. Sendhilkumar, 2016. Prevalence of risk factors for falls among elderly people living in long-term care homes. J. Clin. Gerontol. Geriatr., 7: 99-103.

Hwang, S.H. and S.B. Pan, 2016. Fall detection system using the open source hardware and RGB camera. J. Korean Institute Inform. Technol., 14: 19-24.

Igual, R., C. Medrano and I. Plaza, 2013. Challenges, issues and trends in fall detection systems. Biomed. Eng. Online, Vol. 12, 10.1186/1475-925X-12-66.

Kerr, M. and R. van Schyndel, 2014. Adapting law enforcement frameworks to address the ethical problems of CCTV product propagation. IEEE Secur. Privacy, 12: 14-21.

Kim, H., I. Kim and J. Kim, 2015. Designing the smart foot mat and its applications: As a user identification sensor for smart home scenarios. Adv. Sci. Technol. Lett., 87: 1-5.

Klenk, J., C. Becker, F. Lieken, S. Nicolai and W. Maetzler et al., 2011. Comparison of acceleration signals of simulated and real-world backward falls. Med. Eng. Phys., 33: 368-373.

Kumar, V., B.C. Yeo, W.S. Lim, J.E. Raja and K.B. Koh, 2018. Development of electronic floor mat for fall detection and elderly care. Asian J. Sci. Res., 11: 344-356.

Lu, K.L. and E.T.H. Chu, 2018. An image-based fall detection system for the elderly. Appl. Sci., Vol. 8, No. 10. 10.3390/app8101995.

Ozdemir, A.T. and B. Barshan, 2014. Detecting falls with wearable sensors using machine learning techniques. Sensors, 14: 10691-10708. 
Romli, M.H., L. Mackenzie, M. Lovarini and M.P. Tan, 2016. Pilot study to investigate the feasibility of the Home Falls and Accidents Screening Tool (HOME FAST) to identify older Malaysian people at risk of falls. BMJ Open, Vol. 6, No. 8. 10.1136/bmjopen2016-012048

Saenz-Cogollo, J.F., M. Pau, B. Fraboni and A. Bonfiglio, 2016. Pressure mapping mat for tele-home care applications. Sensors, Vol. 16. 10.3390/s16030365

Shibuya, N., B.T. Nukala, A.I. Rodriguez, J. Tsay, T.Q. Nguyen, S. Zupancic and D.Y. Lie, 2015. A real-time fall detection system using a wearable gait analysis sensor and a Support Vector Machine (SVM) classifier. Proceedings of the 2015 8th International Conference on Mobile Computing and Ubiquitous Networking (ICMU'15), January 20-22, 2015, IEEE, Hakodate, Japan, pp: 66-67.
WHO., 2008. WHO Global Report on Falls Prevention in Older Age. World Health Organization, Geneva, Switzerland, ISBN: 978-92-4-156353-6, Pages: 47.

Wilding, M.J., L. Seegert, S. Rupcic, M. Griffin, S. Kachnowski and S. Parasuraman, 2013. Falling short: Recruiting elderly individuals for a fall study. Ageing Res. Rev., 12: 552-560.

Xu, J., X. Liu, Z. Huo, C. Deng, F. Nie and H. Huang, 2017. Multi-class support vector machine via maximizing multi-class margins. Proceedings of the 26th International Joint Conference on Artificial Intelligence (IJCAI'17), August 19-25, 2017, AAAI Press, Melbourne, Australia, pp: 3154-3160.

Yu, M., A. Rhuma, S.M. Naqvi, L. Wang and J. Chambers, 2012. A posture recognition-based fall detection system for monitoring an elderly person in a smart home environment. IEEE Trans. Inform. Technol. Biomed., 16: 1274-1286. 